International Journal of Computer Networks \& Communications (IJCNC) Vol.4, No.6, November 2012

\title{
UNIFIED ANALYSIS OF TWO-HOP COOPERATIVE AMPLIFY-AND-FORWARD MULTI-RELAY NETWORKS
}

\begin{abstract}
This article develops an extremely simple and tight closed-form approximation for the moment generating function (MGF) of signal-to-noise ratio (SNR) for two-hop amplify-and-forward relayed paths over generalized fading environments. The resulting expression facilitates efficient analysis of twohop cooperative amplify-and-forward $(C A F)$ multi-relay networks over a myriad of stochastic channel models (including mixed-fading scenarios where fading statistics of distinct links in the relayed path may be from different family of distributions). The efficacy of our proposed MGF expression for computing the average symbol error rate (ASER), outage probability, and the ergodic capacity (with limited channel side-information among cooperating nodes) is also studied. Numerical results indicate that the proposed MGF expression tightly approximates the exact MGF formulas and outperforms the existing MGF of lower and upper bounds of the half-harmonic mean (HM) SNR, while overcoming the difficulties associated in deriving an accurate MGF formula for the end-to-end SNR over generalized fading channels. Further application of our new closed-form formula for the MGF of end-to-end SNR for evaluating the average bit and/or packet error rate with adaptive discrete-rate modulation in CAF relay networks is also discussed.
\end{abstract}

Oluwatobi Olabiyi $^{1}$ and Annamalai Annamalai ${ }^{2}$

Centre of Excellence for Communication Systems Technology Research

Department of Electrical and Computer Engineering

Prairie View A\&M University, Texas 77446

${ }^{1}$ engr3os@gmail.com ${ }^{2}$ aaannamalai@pvamu.edu

\section{KEYWORDS}

cooperative diversity, average symbol error probability, outage probability, ergodic capacity, moment generating function approach

\section{INTRODUCTION}

The broadcast nature of wireless transmissions has enabled a new communication paradigm known as "cooperative communications" wherein the source node communicates with the destination node with the help of one or more relay nodes to harness the inherent spatial diversity gain in wireless networks without requiring multiple transceivers at the destination node. It is an active and growing field of research because this form of "user cooperation diversity" has the ability to overcome the practical implementation issue of packing a large number of antenna elements on small-sized hand-held portable wireless devices and sensor nodes, besides enabling the source node to tap into the available resources of local neighbouring nodes to increase its throughput, range, reliability, and covertness.

Cooperative diversity can be broadly categorized as one of amplify-and-forward, decode-andforward, and compress-and-forward relaying strategies, each corresponding to different protocol implementations at the relay nodes [1]-[2]. Other variations cooperative diversity 
International Journal of Computer Networks \& Communications (IJCNC) Vol.4, No.6, November 2012

strategies include opportunistic, incremental, variable-gain and fixed-gain (either blind or semiblind) relaying that are based on the availability of channel side information (CSI) and the number of nodes actively participating in information relaying [1]-[4]. In this article, we primarily focus on variable-gain cooperative amplify-and-forward (CAF) relaying strategy although the analysis may be extended to other categories and variations of cooperative relaying strategies.

While numerous performance metrics of CAF relay networks have been considered in the literature including ergodic/outage capacity, outage probability, and ASER (see [5]-[25] and references therein), most results in the literature were restricted to either Rayleigh or Nakagami-m channels, or the authors' resort to asymptotic analysis, or develop performance bounds. In fact, determination of the exact performance of CAF multi-relay networks over a generalized fading environment with independent but non-identically distributed (i.n.d) fading statistics via an analytical approach is known to be a daunting task. This is attributed to the difficulty in deriving the exact probability density function (PDF) or the moment generating function (MGF) of the end-to-end signal-to-noise ratio (SNR). For instance, the exact MGF expression for the desired SNR over i.n.d Nakagami-m derived recently in [14] involves triple summation terms involving $k^{\text {th }}$ derivative of the product of Whittaker functions with complicated arguments, which is not easily evaluated using a general computing platform, besides being restrictive to positive integer fading index $m$. Other "exact" formulas (based on the half-harmonic mean (HM) tight bound of exact end-to-end SNR) for the PDF or the MGF of SNR in CAF relay networks can be found in [5]-[7] (for Rayleigh fading), [8] (for Nakagami-m environment with independent and identically distributed (i.i.d) fading statistics) and [16]. Although the development in [16] is interesting and their MGF approach can be applied to a wide range of fading environments, the resulting integral expressions are often too complicated to compute or very time-consuming (due to the need to evaluate a nested two-fold integral term with complicated arguments that includes infinite series in some cases). To circumvent this difficulty, some authors have developed bounds for the half-harmonic mean (HM) MGF of end-to-end SNR of CAF multi-relay networks in Rayleigh [6][10], Nakagami-m [11][12] and Rice [13] fading environments. In [9], Ribeiro et. al. developed an asymptotic expression for multi-relay CAF diversity system that employs BPSK modulation in Rayleigh and Rice fading channels (although all their results were limited to only Rayleigh channels) using an asymptotic analysis technique similar to that developed in [26] and [27] for noncooperative diversity systems. In [15], the asymptotic analysis result of [9] was extended to a Nakagami-m fading channel.

In this article, we develop a new unified approximate MGF expression for the SNR of two-hop relayed path which is then used to derive a tight approximate MGF of end-to-end SNR for multi-relay networks. Unlike the contributions from related works found in the literature, our closed-form MGF formula requires only the knowledge of the MGF of SNR of individual links, which makes it readily applicable to mixed fading and composite multipath/shadowing (e.g., Suzuki distribution, $K$-distribution, $G$-distribution, etc.) environments. The efficiency and accuracy of our proposed solution is compared to existing closed-form and/or integral expressions (when available) to demonstrate its utility and versatility. Several important performance metrics of 2-hop CAF relay networks are considered such as average symbol error rate (ASER), outage probability and ergodic capacity. In addition, our MGF expression may be exploited for efficient evaluation of ASER and/or average packet error rate (APER) with discrete-rate adaptive modulation and/or computation of the average detection probability of relay-assisted energy detector over generalized fading channel. Numerical results indicate that 
International Journal of Computer Networks \& Communications (IJCNC) Vol.4, No.6, November 2012

our MGF expression is much closer to the exact MGF compared to the widely used upper and lower bounds for the MGF of half harmonic mean SNR (e.g., [10]-[13]) despite its simplicity and generality. While our unified MGF formula is slightly less accurate compared to the MGF of the half-harmonic mean SNR (only available for specific fading scenarios) in [5]-[8] and [16], it is still very close to the exact MGF expression derived in [14] and [18] (also available for only specific fading environments) while ensuring numerically stable and low computational cost. In fact, our proposed solution is perhaps the only accurate closed-form MGF expression that can effectively capture the independent and non-identical distributed (i.n.d) fading statistics across distinct wireless links of a route path in a unified manner. It is important to note that although the mathematical framework developed in [16] is applicable to generalized fading channels, it is quite cumbersome and/or numerically unstable for computing the MGF of end-to-end SNR in certain cases such as Rice and Nakagami-q environments because it requires the evaluation of an integral whose integrand is a product of infinite series containing Bessel functions with complicated arguments.

Moreover, for the specific case of ASER analysis of CAF relay networks, we express the final ASER in closed-form (i.e., as a weighted sum of MGF of end-to-end SNR). This is accomplished by using the second-order exponential approximation for the conditional error probability (CEP) of M-ary phase shift keying (MPSK) and/or M-ary quadrature amplitude modulation (MQAM) digital modulation schemes (e.g., $P_{s}(\varepsilon \mid \gamma) \approx a e^{-b \gamma}+c e^{-2 b \gamma}$ ) derived in [19].

The resulting "unified" ASER expressions are much more general, and more accurate over a wide range of channel SNRs (especially at larger values of fading severity index than the corresponding asymptotic ASER formulas presented in [9] and [15] while ensuring a low computational cost for evaluating the desired ASER (since they are in closed-form). It is also important to highlight that the simplicity of our final approximate ASER formula may facilitate further system level optimization tasks (e.g., optimal power assignment and/or relay placement in CAF multi-relay networks) although such investigations are beyond the scope of this article.

The remainder of this paper is organized as follows. In Section II, we briefly review the system model and discuss the key steps in our development of a tight approximation for the MGF of end-to-end SNR. Several applications of our proposed MGF formula are discussed in Section III along with selected numerical results to highlight its utility (e.g., comparisons with related results in the literature) followed by some concluding remarks in Section IV.

\section{Tight Approximation fOR the MGF of SNR in CAF Relay NETWORKS}

Consider a cooperative wireless network model that comprises of a source node $S$ which communicates with a destination node $D$ via a direct-link and through $N$ amplify-and-forward relays, $R_{i}, i \in\{1,2, \ldots, N\}$, in two transmission phases. During the initial Phase I, $S$ broadcasts a signal to $D$ and to the relays $R_{i}$, where the channel fading coefficients between $S$ and $D, S$ and the $i$-th relay node $R_{i}, R_{i}$ and $D$ are denoted by $h_{s, d}, h_{s, i}$ and $h_{i, d}$ respectively. During the second phase of cooperation, each of the $N$ relays transmits the received signal after amplification via orthogonal transmissions. If a maximum ratio combiner (MRC) is employed at the destination node $D$ to coherently combine all the signals received during these two transmission phases, the effective end-to-end SNR is given by [1], [10]-[14], 
International Journal of Computer Networks \& Communications (IJCNC) Vol.4, No.6, November 2012

$\gamma_{T}=\gamma_{s, d}+\sum_{i=1}^{N} \frac{\gamma_{s, i} \gamma_{i, d}}{\gamma_{s, i}+\gamma_{i, d}+c}=\gamma_{s, d}+\sum_{i=1}^{N} \gamma_{i}$

where $c$ is a constant (i.e., assumes the value of 1 for the exact end-to-end SNR and 0 for halfharmonic mean ${ }^{1}$ bound), $\gamma_{a, b}=\left|h_{a, b}\right|^{2} E_{s} / N_{o}$ corresponds to the instantaneous SNRs of link $a-b$, $E_{s}$ denotes the average symbol energy and $N_{0}$ corresponds to the noise variance. Hence the MGF of end-to-end SNR shown in (1) can be expressed as

$\phi_{\gamma_{T}}=\phi_{\gamma_{s, d}}(s) \prod_{i=1}^{N} \phi_{\gamma_{i}}(s)$

The MGF of the direct link $\phi_{\gamma_{s, d}}(s)$ is a single channel and can be easily obtained in the literature (e.g., [28, Table 2.2]). However, it is well-known in the literature that evaluation of the MGF of SNR for the relayed path $\gamma_{i}=\frac{\gamma_{s, i} \gamma_{i, d}}{\gamma_{s, i}+\gamma_{i, d}+c}$ is quite challenging and the exact MGF formula $(c=1)$ only exists for Nakagami-m fading channel with integer fading severity index, viz., [14, Eq. (7)]

$$
\phi_{\gamma_{i}}^{(E)}(s)=1-2 s \sum_{n=0}^{m_{s, j}-1} \sum_{k=0}^{m_{i, d}-1} \sum_{l=1}^{k} C_{1}(n, k, l) \sum_{q=0}^{n+l+2}\left(\begin{array}{c}
n+l+2 \\
q
\end{array}\right) \Psi_{1}(n, k, l, q)
$$

where

$$
\Psi_{1}(n, k, l, q)=\frac{\sqrt{\beta_{1} \beta_{2}} \Gamma(l+1)}{2(-1)^{m_{s, d}+k-q+1}} \frac{d^{m_{s, d}+k-q+1}}{d t^{m_{s, d}+k-q+1}}\left[\left.e^{\frac{t}{2}} W_{-\frac{n+1+2}{2}, \frac{n-1+2}{2}}\left(\frac{t-\sqrt{t^{2}-\frac{4}{\beta_{1} \beta_{2}}}}{2}\right) W_{\frac{n++2,2}{2}, \frac{n-1+2}{2}}\left(\frac{t+\sqrt{t^{2}-\frac{4}{\beta_{1} \beta_{2}}}}{2}\right)\right|_{t=s+\frac{1}{\beta_{1}}+\frac{1}{\beta_{2}}},\right.
$$

$\beta_{1}=\Omega_{s, i} / m_{s, i}, \beta_{2}=\Omega_{i, d} / m_{i, d}, C_{1}=\frac{\beta_{1}^{\left(n-l-2 m_{s, d}\right) / 2} \beta_{2}^{(l-n-1-2 k) / 2}}{l !(k-l) ! n !\left(m_{s, d}-n-1\right) !}, m_{a, b}$ and $\Omega_{a, b}$ are the Nakagami-m

fading index and average received SNR of wireless link $a-b$, respectively, and $W(.,$.$) denotes$ the Whittaker function [29]. Although Eq. (3) has only finite summation terms, it involves the evaluation of $k^{\text {th }}$ derivatives of product of Whitaker functions which is not necessarily a trivial task (i.e., one may have to resort to a suitable computing platform such as MAPLE software to compute the above MGF using a symbolic differentiation tool). Due to this limitation, several researchers have considered a more tractable MGF for half-harmonic mean SNR $\gamma_{i}$ (i.e., $c=0$ ) which has been shown to be very accurate at moderate and high SNR [5]-[8]. Even in this case, the obtained closed-form results are still limited to i.n.d Rayleigh [5]-[7] (seemingly different expressions but numerically same), i.i.d [8] and i.n.d Nakagami-m channels (for positive integer fading index $m$ ) [14]. These results are summarized in (4)-(8) for readers' convenience.

Rayleigh Fading: [4, Eq. (20)], [5, Eq. (7)], [6, Eq. (52)]

\footnotetext{
${ }^{1}$ Most prior work computes the PDF or MGF of $\gamma_{i}=\gamma_{s, i} \gamma_{k, d} /\left(\gamma_{s, i}+\gamma_{i, d}\right)$ which becomes accurate for moderate and large SNR values.
} 
International Journal of Computer Networks \& Communications (IJCNC) Vol.4, No.6, November 2012

$$
\phi_{\gamma_{i}}^{(H M)}(s)=\frac{16}{3 \Omega_{s, i} \Omega_{i, d}\left(A_{1}+s\right)^{2}}\left[\frac{4\left(\frac{1}{\Omega_{s, i}}+\frac{1}{\Omega_{i, d}}\right)}{\left(A_{1}+s\right)}{ }_{2} F_{1}\left(3, \frac{3}{2} ; \frac{5}{2} ; \frac{A_{2}+s}{A_{1}+s}\right)+{ }_{2} F_{1}\left(2, \frac{1}{2} ; \frac{5}{2} ; \frac{A_{2}+s}{A_{1}+s}\right)\right]
$$

where ${ }_{2} F_{1}(., . ; . ;$.$) is the Gauss Hypergeometric function [29], A_{1}=\frac{1}{\Omega_{s, i}}+\frac{1}{\Omega_{i, d}}+\frac{2}{\sqrt{\Omega_{s, i} \Omega_{i, d}}}$,

$$
\begin{aligned}
A_{2}= & \frac{1}{\Omega_{p, i}}+\frac{1}{\Omega_{i, d}}-\frac{2}{\sqrt{\Omega_{p, i} \Omega_{i, d}}}, \\
\phi_{\gamma_{i}}^{(H M)}(s) & =\frac{\frac{4 p(f(s)-\sigma) \sqrt{f^{2}(s)}}{f(s) \sqrt{4 p-f^{2}(s)}} a \cos \left(\frac{f(s)}{2 \sqrt{p}}\right)+\sigma f(s)-4 p}{f^{2}(s)-4 p}, \quad \operatorname{Re}\{s\}<\sigma / p
\end{aligned}
$$

where $f(s)=\sigma-p s, \sigma=\Omega_{s, i}+\Omega_{i, d}, p=\Omega_{s, i} \Omega_{i, d}$,

$$
\phi_{\gamma_{i}}^{(H M)}(s)=\left[\left(1 / \Omega_{s, i}-1 / \Omega_{i, d}\right)^{2}+\left(1 / \Omega_{s, i}-1 / \Omega_{i, d}\right) s\right] / \Delta^{2}+\frac{2 s}{\Delta^{3} \Omega_{s, i} \Omega_{i, d}} \ln \left(\left(s+\Delta+\frac{1}{\Omega_{s, i}}+\frac{1}{\Omega_{i, d}}\right)^{2} \frac{\Omega_{s, i} \Omega_{i, d}}{4}\right)
$$

where $\Delta=\sqrt{\left(1 / \Omega_{s, i}-1 / \Omega_{i, d}\right)^{2}+2 s\left(1 / \Omega_{s, i}+1 / \Omega_{i, d}\right)+s^{2}}$.

Nakagami-m Fading: [8, Eq. (26)], [14, Eq. (8)]

$$
\phi_{\gamma_{i}}(s)={ }_{2} F_{1}\left(m, 2 m ; m+\frac{1}{2} ; \frac{-\Omega_{i}}{4 m}\right)
$$

where $\Omega_{i}=\Omega_{s, i}=\Omega_{i, d}$ corresponds to the average link SNR of each i.i.d wireless link in a relayed path.

$$
\begin{aligned}
\phi_{\gamma_{i}}^{(H M)}(s) & =1-2 s \sum_{n=0}^{m_{s, i}-1} \sum_{k=0}^{m_{i, d}-1} \sum_{l=1}^{k} C_{1}(n, k, l) \Psi_{2}(n, k, l) \\
\Psi_{2}(n, k, l) & =\frac{\sqrt{\pi} \Gamma\left(m_{s, d}+k+n-l+2\right) \Gamma\left(m_{s, d}+k-n+l\right)}{\Gamma\left(m_{s, d}+k+\frac{3}{2}\right)}\left(\frac{16}{\beta_{1} \beta_{2}}\right)^{(n-l+1) / 2} \\
& \times \frac{{ }_{2} F_{1}\left(m_{s, d}+k+n-l+2, n-l+\frac{3}{2} ; m_{s, d}+k+\frac{3}{2} ; \bar{s}\right)}{\left(s+\left(\sqrt{1 / \beta_{1}}+\sqrt{1 / \beta_{2}}\right)^{2}\right)^{m_{s, d}+k+n-l+2}}, \bar{s}=\frac{s+\left(\sqrt{1 / \beta_{1}}-\sqrt{1 / \beta_{2}}\right)^{2}}{s+\left(\sqrt{1 / \beta_{1}}+\sqrt{1 / \beta_{2}}\right)^{2}}, \text { and } \beta_{1},
\end{aligned}
$$

$\beta_{2}$ and $C_{1}$ are defined as in (3).

It is also evident from Eq. (9) that the generalized MGF formula developed in [16] requires an evaluation of a double nested integral, viz., [16, Eq. (5)]

$$
\phi_{\gamma_{i}}^{(H M)}(s)=1-\sqrt{s} \int_{0}^{\pi / 2} \frac{\sec ^{2}(\xi)}{\sqrt{\tan (\xi)}} J_{1}(2 \sqrt{s \tan (\xi)}) \phi_{1 / \gamma_{i}}(\tan (\xi)) d \xi
$$


International Journal of Computer Networks \& Communications (IJCNC) Vol.4, No.6, November 2012

where $\phi_{1 / \gamma_{i}}(s)=\prod_{n \in\{(s, i),(i, d)\}}\left[1-\sqrt{s} \int_{0}^{\pi / 2} \frac{\sec ^{2}(\xi)}{\sqrt{\tan (\xi)}} J_{1}(2 \sqrt{s \tan (\xi)}) \phi_{\gamma_{n}}(\tan (\xi)) d \xi\right]$ and $J_{1}($.$) denotes the$ first-order Bessel function of the first kind. Although the above expression is tractable for Rayleigh and Nakagami- $m$ channels (e.g., when $\phi_{1 / \gamma_{i}}(s)$ is available in closed-form), it can be quite tedious or numerically unstable for certain fading environment (e.g., Rice and Nakagami$q$ fading environments). This is because $\phi_{1 / \gamma_{i}}(s)$ is expressed as an infinite series consisting of special functions (e.g. Bessel function) with complicated arguments.

In order to further improve the mathematical tractability, bounds like $\gamma_{i}^{(L B)} \leq \gamma_{i}^{H M} \leq \gamma_{i}^{(U B)}$ where $\gamma_{i}^{(L B)}=\frac{1}{2} \min \left(\gamma_{s, i}, \gamma_{i, d}\right)$ and $\gamma_{i}^{(U B)}=\min \left(\gamma_{s, i}, \gamma_{i, d}\right)$ have also been developed to minimize the complexity of the problem in Nakagami-m [11], [12] and Rice channels with i.n.d diversity paths [13]. These expressions are summarized in (10) and (11), respectively.

Nakagami-m Fading: [12, Eq. (11)]

$$
\phi_{\gamma_{i}}^{(U B)}(s)=\sum_{\substack{k \in\{(s, i),(i, \mathrm{~d})\} \\ j \neq k}} \frac{\Gamma\left(m_{k}+m_{j}\right)}{\Gamma\left(m_{k}\right) \Gamma\left(m_{j}\right)}\left(\frac{\Omega_{j} m_{k}}{s \Omega_{j} \Omega_{k}+\Omega_{j} m_{k}+\Omega_{k} m_{j}}\right) \times \frac{1}{m_{k}}{ }_{2} F_{1}\left(1-m_{j}, m_{k} ; 1+m_{k} ; \frac{\left(s \Omega_{k}+m_{k}\right) \Omega_{j}}{s \Omega_{j} \Omega_{k}+\Omega_{j} m_{k}+\Omega_{k} m_{j}}\right)
$$

Rice Fading: [13, Eq. (10)]

$$
\phi_{\gamma_{i}}^{(U B)}(s)=\sum_{\substack{k \in\{(s, i)(i, d)\} \\ j \neq k}} 2 A_{k} e^{-K_{k}} \vartheta\left(\sqrt{2 A_{j}}, \sqrt{2 K_{j}}, \sqrt{2 A_{k} K_{k}}, 2\left(s+A_{k}\right)\right)
$$

where $A_{i}=\left(1+K_{i}\right) / \Omega_{i}, \quad K_{a, b}$ denotes the Rice factor of wireless link $a-b, I_{0}($.$) is the modified$ Bessel function of the first kind and $\vartheta(a, b, c, d)=\frac{1}{d} e^{\frac{c^{2}}{2 d}} Q\left(b \sqrt{\frac{d}{\left(d+a^{2}\right)}}, \frac{a c}{\sqrt{d\left(d+a^{2}\right)}}\right)-\frac{a^{2}}{d\left(d+a^{2}\right)} e^{\frac{c^{2}-b^{2} d}{2\left(d a^{2}\right)}} I_{0}\left(\frac{a b c}{\left(d+a^{2}\right)}\right)$.

The corresponding MGF of the lower bound of HM SNR of relay path can be obtained by replacing $s$ in (10) and (11) by $s / 2$, i.e. $\phi_{\gamma_{i}}^{(L B)}(s)=\phi_{\gamma_{i}}^{(U B)}(s / 2)$.

It should be evident by now that the evaluation of the MGF of end-to-end SNR even for twohop CAF relay networks can be cumbersome, which limits a comprehensive characterization of cooperative diversity networks over generalized fading environments. This also motivates the development of a computationally efficient MGF formula for $\gamma_{i}$ without compromising on the accuracy over a wide range of channel SNRs. Furthermore, it will be of interest to have an MGF expression that can easily capture the mixed-fading and composite fading environments. This article highlights the derivation of a MGF of end-to-end SNR for CAF relay networks that meets the above attributes. We would also like to point out that although the "asymptotic" MGF of SNR proposed in [17, Eq. (10)] yields a good ASER approximation, that expression is not a valid MGF because its numerical value ranges between 0 and 2 (instead of between 0 and 1 ). Besides, a rigorous proof for the derivation of the asymptotic MGF is lacking. These issues are also addressed in this article. Specifically, we derive a very tight approximation for the MGF of $\gamma_{i}$ (hereafter, referred to as $\left.\phi_{\gamma_{i}}^{(A)}(s)\right)$ which possesses all the attributes of a valid MGF formula.

Let us first consider a CAF diversity network with a single cooperative relay $(N=1)$. In this case, the end-to-end SNR (with MRC combining of signal replicas at the destination node) is given by 
International Journal of Computer Networks \& Communications (IJCNC) Vol.4, No.6, November 2012

$$
\gamma_{T}=\gamma_{s, d}+\frac{\gamma_{s, 1} \gamma_{1, d}}{1+\gamma_{s, 1}+\gamma_{1, d}}=\gamma_{s, d}+\gamma_{1}
$$

In the following, we will derive a tight approximation for the MGFs of $\gamma_{1}$ and $\gamma_{T}$.

Proposition: Let us consider four non-negative independent random variables $W, X, Y$ and $Z$ with PDFs $f_{\kappa}()$, CDFs $F_{\kappa}($.$) , and MGFs \phi_{\kappa}(),. \kappa \in\{W, X, Y, Z\}$. If we define $W=\frac{X Y}{X+Y+1}$ and $Z=\min (X, Y)$, then the MGF of $W$ can be approximated as

$$
\phi_{W}(s) \approx \phi_{X}(s)+\phi_{Y}(s)-\phi_{X}(s) \phi_{Y}(s)
$$

Before providing a proof for (13), we prove the following two lemmas for the CDF of $W$.

Lemma 1: The CDF of $W=\frac{X Y}{X+Y+1}$ is given by

$$
F_{W}(w)=F_{Y}(w)+\int_{w}^{\infty} F_{X}\left(\frac{w(y+1)}{y-w}\right) f_{Y}(y) d y
$$

Proof: See Appendix A.

Lemma 2: A tight lower bound for the CDF and MGF of $W$ are given by (13) and (14), respectively:

$$
\begin{aligned}
F_{W}(w) & \geq F_{X}(w)+F_{Y}(w)-F_{X}(w) F_{Y}(w) \\
\phi_{W}(s) & \geq \phi_{Z}(s)
\end{aligned}
$$

where $\phi_{Z}(s)=\phi_{X}(s)+\phi_{Y}(s)-\int_{0}^{\infty} e^{-s x}\left[f_{X}(x) F_{Y}(x)+f_{Y}(x) F_{X}(x)\right] d x$.

Proof: See Appendix B.

Thus, the exact MGF of $\gamma_{T}$ in (1) can be tightly approximated (using Eq. (13)) as

$$
\phi_{\gamma_{i}}^{(E)}(s) \approx \phi_{\gamma_{i}}^{(A)}(s)=\phi_{\gamma_{s, d}}\left(\phi_{\gamma_{s, 1}}(s)+\phi_{\gamma_{1, d}}(s)-\phi_{\gamma_{s, 1}}(s) \phi_{\gamma_{1, d}}(s)\right)
$$

since $\gamma_{s, d}$ and $\gamma_{1}$ are assumed to be independent random variables (i.e., a reasonable assumption because the nodes are spatially distributed over a large geographical area).

It is also important to highlight that the new approximate MGF expression (B.5) is much more compact and can be easily generalized to more sophisticated channel models (including mixedfading scenarios and shadowing) compared to (B.4) (corresponding to the MGF of $\left.\gamma_{k}^{(U B)}=\min \left(\gamma_{s, k}, \gamma_{k, d}\right)\right), \phi_{\gamma_{i}}^{(E)}(s)$, and $\phi_{\gamma_{i}}^{(H M)}(s)$ (i.e., can be derived from (A.1)) which may not necessarily yield a closed-form expression in a generalized fading environment. Besides, it only requires the knowledge of MGF of individual links in closed-form, which is readily available in the literature [28, Table 2.2, pp. 21].

It is also straight-forward to generalize (17) to multi-relay CAF networks. In this case, the approximate MGF of $\gamma_{T}$ as shown in (2) is given by 
International Journal of Computer Networks \& Communications (IJCNC) Vol.4, No.6, November 2012

$$
\phi_{\gamma_{T}}^{(E)}(s) \approx \phi_{\gamma_{T}}^{(A)}(s)=\phi_{\gamma_{s, d}}(s) \prod_{i=1}^{N}\left(\phi_{\gamma_{s, i}}(s)+\phi_{\gamma_{i, d}}(s)-\phi_{\gamma_{s, i}}(s) \phi_{\gamma_{i, d}}(s)\right)
$$

Fig. 1 shows a comparison of the exact, approximate, HM bound, upper and lower HM bound MGFs of SNR for a two-hop relayed path over i.i.d and i.n.d Nakagami- $m$ channel. The curve corresponding to MGF of $\gamma_{1}^{(H M)}$ is generated using the HM MGF given in (7) [8, Eq. (26)] and [16, Eq. (5) and Table 1] for Nakagami- $m$ fading channels. The corresponding MGF of $\gamma_{1}^{(U B)}=\min \left(\gamma_{s, 1}, \gamma_{1, d}\right)$ (i.e., can be evaluated using (B.4)) and $\gamma_{1}^{(L B)}=\gamma_{1}^{(U B)} / 2$ are given in [12, Eqs. (9) - (11)]. We observe at a very small mean SNR value, the exact MGF is closer to the lower bound (LB) than any other curves. However as the mean SNR and/or channel fading index increases, the exact end-to-end SNR approaches the HM and upper bound (UB) of HM, as expected. More importantly, at higher SNR, our approximation is much closer to the exact and HM MGF of $\gamma_{1}$ compared to the bounds (MGF of $\gamma_{1}^{(L B)}$ or $\gamma_{1}^{(U B)}$ ) over a wide range of its argument $s$. We would also like to point out that the MGF of HM SNR in (4)-(8) [5]-[8] and [14] are channel specific (i.e., limited to only i.n.d. Rayleigh and i.n.d Nakagami-m channels) while the corresponding MGF formula in (9) [16] is more general, but it involves the computation of a nested double integral term, and may be numerically unstable or too complex for some fading environments (e.g., Rice fading). On the contrary, our approximation (18) is both simple and can readily handle complex fading channel models. In fact, it requires only the knowledge of MGF of individual link SNRs, which are readily available in closed-form for a myriad of stochastic channel models (see [28, Table 2.2, pp. 21]). In Section III, we will highlight the utility of our new MGF formula for efficient analysis of multi-relay CAF networks and computation of the average detection probability in relay-assisted spectrum sensing application.

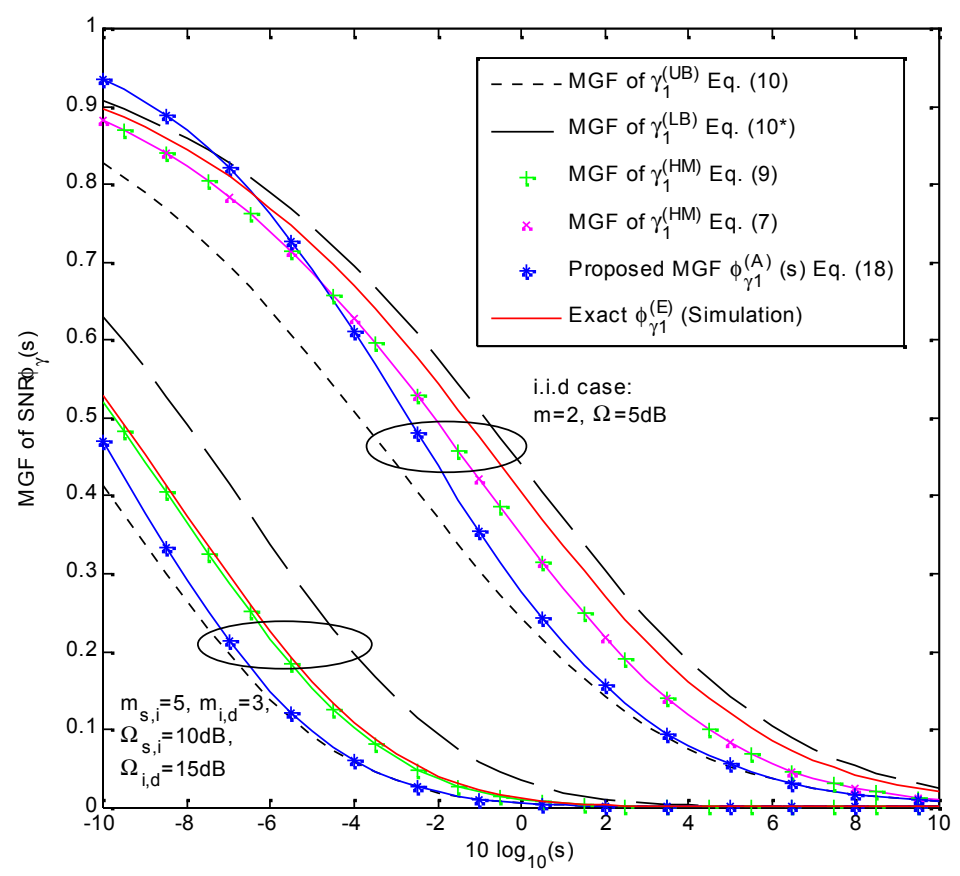

Fig. 1 Comparison between the exact, approximate and bounds for the MGF of effective SNR of relay path over i.i.d and i.n.d Nakagami-m channels; (Eq.(10*) indicates evaluation by replacing $s$ in (10) by $s / 2)$. 
International Journal of Computer Networks \& Communications (IJCNC) Vol.4, No.6, November 2012

\section{APPlications of ClOSED-Form MGF OF END-To-END SNR IN CAF RELAY NETWORKS}

\subsection{Unified Closed-Form ASER Expression for 2-Hop CAF Multi-Relay Networks}

In general, the ASER of CAF multi-relay networks can be computed by averaging the CEP $P_{s}(\varepsilon \mid \gamma)$ over the PDF of $\gamma_{T}$ as

$$
\overline{P_{s}}=\int_{0}^{\infty} P_{s}(\varepsilon \mid \gamma) f_{\gamma_{T}}(\gamma) d \gamma
$$

If the CEP can be expressed in a "desirable exponential form", then the averaging task in (19) can be simplified dramatically. In this article, we exploit an accurate second-order exponentialtype approximation for the CEP [19] as summarized in Table I for MPSK and MQAM digital modulations with a fixed set of coefficients $a_{1}=0.2938, b_{1}=1.0483$, and $c_{l}=0.5070$. This approximation has been shown to be slightly more accurate than the exponential-type approximations in [17] and [30], and practically same as the exact $Q$-function representation in [31]. If we replace the CEP in (19) with its exponential approximation (as shown in Table I), then (19) simplifies into a closed-form formula (i.e., can be expressed as a weighted sum of MGF of $\gamma_{T}$ alone). For instance, the ASER of CAF multi-relay network with MPSK modulation is given by

$$
\overline{P_{s}}=a_{1} \phi_{\gamma_{T}}\left(b_{1} \sin ^{2}(\pi / M)\right)+c_{1} \phi_{\gamma_{T}}\left(2 b_{1} \sin ^{2}(\pi / M)\right)
$$

where $\phi_{\gamma_{T}}($.$) is defined in (18). Similar expressions can be obtained easily for other digital$ modulation schemes (i.e., MQAM). It should be emphasized that there are two sources of approximation errors in our unified closed-form ASER expression (20), with the first stemming from the approximation of the MGF of SNR in (18) and the other from CEP approximation via $\operatorname{erfc}(x)=a_{1} e^{-b_{1} x^{2}}+c_{1} e^{-2 b_{1} x^{2}}$. Even with these approximation errors, we will show that (20) still performs better than the ASER obtained using the upper/lower bounds for the MGF of end-toend SNR (i.e., Eqs. (B.4), (10)-(11)) in conjunction with an exact single finite-range integral expression for the CEP or asymptotic analysis [9][15].

In Fig. 2, the ASER of MQAM with our proposed approximation (using (18) in conjunction with (20)) is compared to the finite-range integral expression using the HM MGF given in (7) [8, Eq. (26)] in a Nakagami-m channel with i.i.d fading statistics as

$\overline{P_{s}}=\frac{4 k}{\pi} \int_{0}^{\pi / 2} \phi_{\gamma_{T}}\left(\frac{3}{2(M-1) \sin ^{2} \theta}\right) d \theta-\frac{4 k^{2}}{\pi} \int_{0}^{\pi / 4} \phi_{\gamma_{T}}\left(\frac{3}{2(M-1) \sin ^{2} \theta}\right) d \theta$ where $M$ denotes the digital constellation size and $k=(\sqrt{M}-1) / \sqrt{M}$. This figure shows that our approximate ASER formula (in closed-form) yields a very good estimate of the actual ASER performance of the CAF relay network over a wide range of mean channel SNR, different fading severity indices and for different constellation sizes. In fact, our closed-form ASER formula performs slightly better than the upper bound MGF case and much better than the lower bound MGF case (even with additional approximation errors for the CEP in our case). However, the gap between the "HM bound" (i.e., the ASER is still expressed in the form of a finite-range integral) and "approximate" ASER performance curves widens as the channel experience less severe fading. This is mainly attributed to the approximation error in the MGF and not the CEP. Although not shown in this article, we also noticed that a similar trend for MPSK digital modulation. 
International Journal of Computer Networks \& Communications (IJCNC) Vol.4, No.6, November 2012

Table I. Q-Function and Exponential-type Representations of the CEP for MQAM and MPSK Digital Modulations ( $\gamma_{s}$ denotes the symbol SNR while $M$ corresponds to the signal constellation size)

\begin{tabular}{|c|c|c|}
\hline $\begin{array}{c}\text { Modulation } \\
\text { scheme }\end{array}$ & Q-Function Representation of CEP & Exponential Representation of CEP \\
\hline BPSK & $Q\left(\sqrt{2 \gamma_{s}}\right)$ & $0.5 a_{1} e^{-b_{1} \gamma_{s}}+0.5 c_{1} e^{-2 b_{1} \gamma_{s}}$ \\
\hline MPSK & $2 Q\left(\sqrt{2 \gamma_{s}} \sin (\pi / M)\right), \quad M \geq 4$ & $a_{1} e^{-b_{1} \gamma_{s} \sin ^{2}(\pi / M)}+c_{1} e^{-2 b_{1} \gamma_{s} \sin ^{2}(\pi / M)}$ \\
\hline MQAM & $\begin{array}{c}4 k Q\left(\sqrt{3 \gamma_{s} /(M-1)}\right)-4 k^{2} Q^{2}\left(\sqrt{3 \gamma_{s} /(M-1)}\right), \\
\text { where } k=(\sqrt{M}-1) / \sqrt{M}\end{array}$ & $\begin{array}{l}2 k a_{1} e^{-\frac{3}{2} \frac{b_{1} \gamma_{s}}{M-1}}+\left(2 k c_{1}-k^{2} a_{1}^{2}\right) e^{-\frac{3 b_{1} \gamma_{s}}{M-1}} \\
-k^{2} c_{1}^{2} e^{-\frac{6 b_{2} \gamma_{s}}{M-1}}-2 k^{2} a_{1} c_{1} e^{-\frac{9}{2} \frac{b_{1} \gamma_{s}}{M-1}}\end{array}$ \\
\hline
\end{tabular}

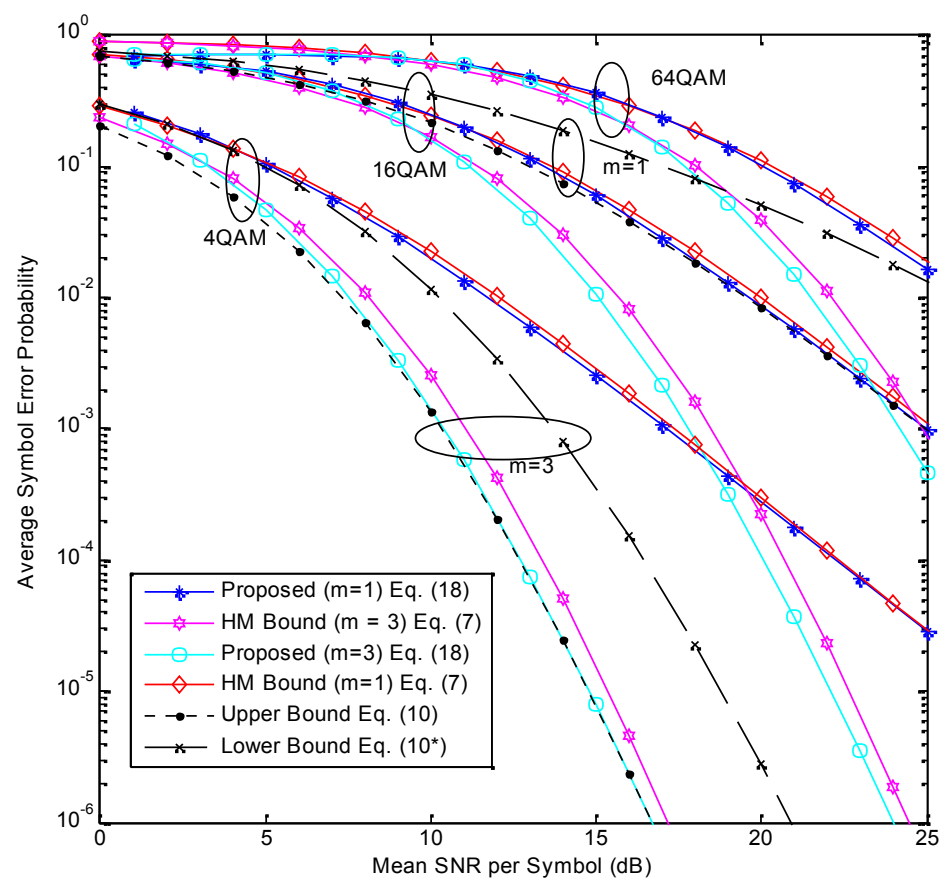

Fig. 2 ASER of MQAM over i.i.d Nakagami channels ( $m=1$ and 3) with single cooperative relay.

Next, we will discuss the efficacy of our new approximation technique for the ASER of CAF networks by comparing it with exact analysis, asymptotic formulas and bounding methods for coherent BPSK modulation (only chosen for illustrative purpose) in Rayleigh and Nakagami- $m$ channels with i.n.d fading statistics via $\overline{P_{s}}=\frac{1}{\pi} \int_{0}^{(M-1) \pi / M} \phi_{\gamma_{T}}\left(\frac{\sin ^{2}(\pi / M)}{\sin ^{2} \theta}\right) d \theta$ with $M=2$ [28]. The closed-form HM i.n.d MGF expression $\phi_{\gamma_{i}}^{(H M)}(s)$ for Rayleigh $(m=1)$ fading is given by (6) [7, Eq. (7)] while the $\phi_{\gamma_{i}}^{(H M)}(s)$ for i.n.d Nakagami-m channel is computed with the aid of (9) [16, Eq. (5) and Table 1]. Bounding technique based on upper and lower bounds of the effective SNR [10]-[13] will also be presented to validate the accuracy of our approach. The MGF of lower bound $\phi_{\gamma_{i}}^{(L B)}(s)$ and upper bound $\phi_{\gamma_{i}}^{(U B)}(s)$ on the effective SNR of two-hop relayed path is obtained from [12, Eq. (11)]. The asymptotic analysis curve is generated using [15 Eq. (15)]. 
International Journal of Computer Networks \& Communications (IJCNC) Vol.4, No.6, November 2012

Fig. 3 provides a comparison between the curves corresponding to all the bounding and asymptotic analyses techniques discussed above over i.n.d Nakagami-m channel ( $m=1$ and 3 ) with two relays. It is apparent that our proposed approximation method performs better than that of the asymptotic analysis in [15] especially at higher values of the fading severity index. Moreover, our approximation (while much closer to the "upper bound" case) performs considerably better than any other approximation or asymptotic analysis at lower SNR regime. Besides, our result (20) is in closed-form.

In Fig. 4, the performance of a CAF relay network (with two cooperating relays) that employ BPSK modulation in Rice (also known as Nakagami-n channel) fading environment is considered. In this case, we noticed that the solution for the HM MGF of SNR for the relayed path presented in [16, Eq. (5) and Table 1] is neither computationally efficient nor numerically stable due to the need to perform numerical integration of product of two infinite series. Thus, the bounding technique [13] and asymptotic analysis [9] are the only viable analytical tools for ASER analysis.

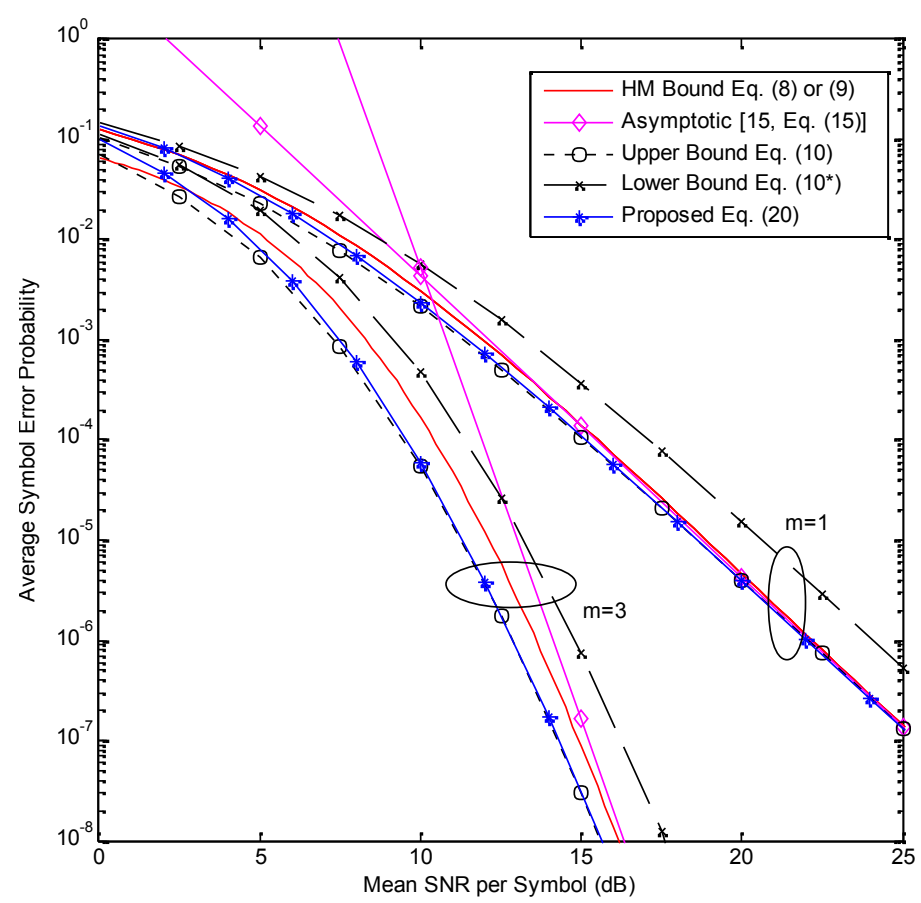

Fig. 3 ASER of BPSK CAF relay networks $(N=2)$ over i.n.d Nakagami-m channels with $\Omega_{s, d}=0.4 \Omega_{T}$, $\Omega_{s, 1}=\Omega_{T}, \Omega_{s, 2}=\Omega_{T}, \Omega_{1, d}=0.43 \Omega_{T}$ and $\Omega_{2, d}=0.43 \Omega_{T}$. 
International Journal of Computer Networks \& Communications (IJCNC) Vol.4, No.6, November 2012

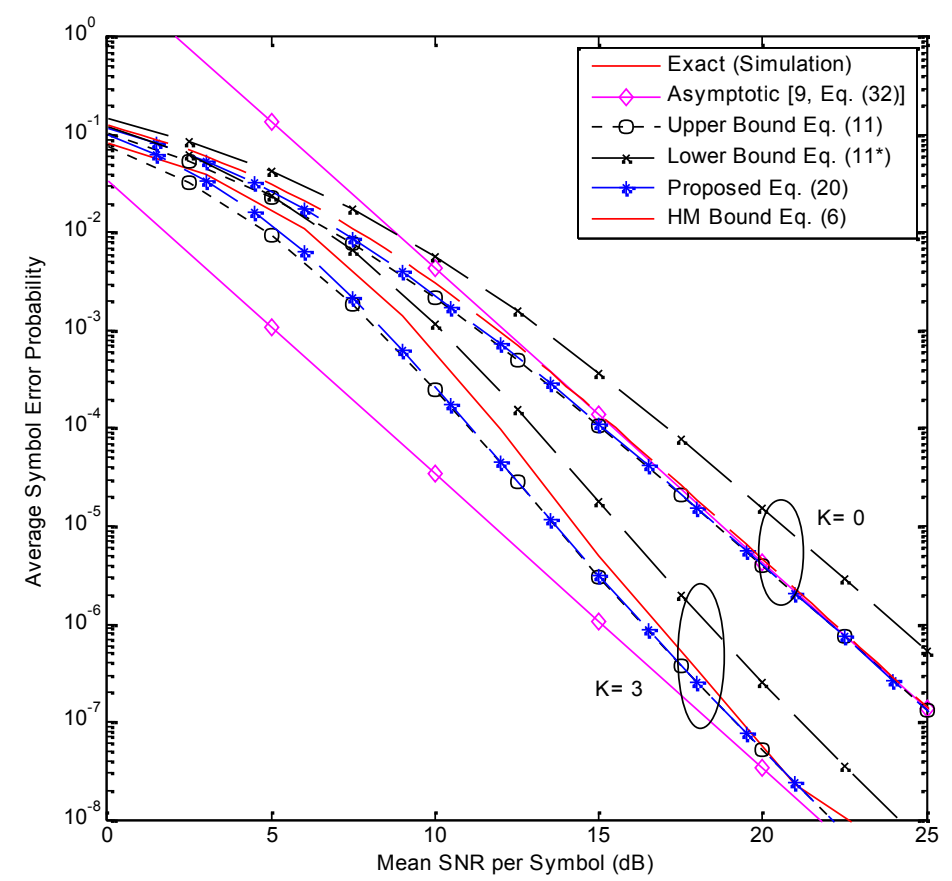

Fig. 4 ASER of BPSK CAF relay networks $(N=2)$ over i.n.d Rice and Rayleigh channels with $\Omega_{s, d}=0.4 \Omega_{T}$, $\Omega_{s, 1}=\Omega_{T}, \Omega_{s, 2}=\Omega_{T}, \Omega_{1, d}=0.43 \Omega_{T}$ and $\Omega_{2, d}=0.43 \Omega_{T}$.

However, in order to provide a comprehensive performance comparison, we also performed Monte Carlo simulation for the ASER of CAF relay system over Rice channel (denoted as "exact" in Fig. 4 for Rice factor $K=3$ ). Closed-form formulas for the MGF of $\gamma_{i}^{(L B)}$ and $\gamma_{i}^{(U B)}$ have been derived in [13] while the asymptotic analysis curve is generated using [9, eq. (32)] (after correcting ${ }^{2}$ a typo). Fig. 4 compares various approximations and bounds for the ASER of CAF relay networks in Rayleigh $(K=0)$ and Rice channels $(K=3)$ with non-identical fading statistics. The general trends (in terms of the tightness of our approximate ASER expression) depicted in Fig. 4 are similar to those observed for the Nakagami-m environment. The asymptotic analysis method [9] or [15] is not very useful in our context since its ASER performance prediction is grossly inadequate in the low SNR regime (for both Rice and Nakagami-m fading channels), although this is the region where cooperative relaying strategies will be typically employed.

\subsection{Outage Probability}

The outage probability $P_{\text {out }}$ is defined as the probability that the instantaneous SNR falls below a specified threshold value $\gamma_{t h}$ and can be calculated using the MGF of SNR as

$$
P_{\text {out }}=\int_{0}^{\gamma_{\text {th }}} \mathfrak{J}^{-1}\left[\phi_{\gamma_{T}}(s)\right] d \gamma=\left.\mathfrak{I}^{-1}\left[\left(\phi_{\gamma_{T}}(s)\right) / s\right]\right|_{\gamma_{\text {th }}}
$$

where $\mathfrak{I}^{-1}$ (.) denotes the inverse Laplace transform operator. In fact, (21) can be evaluated efficiently using numerical methods as discussed in [32]-[33]. However, if closed-form solution

\footnotetext{
${ }^{2}$ In [9, Eq. (33)], the exponential term $e^{-(N+1) K}$ was missing and this explains why only the Rayleigh fading case $(K=0)$ can be evaluated correctly.
} 
International Journal of Computer Networks \& Communications (IJCNC) Vol.4, No.6, November 2012

is desired, our MGF expression in (18) also facilitates the analytical Laplace inversion for some certain fading environments. As an example, consider a single CAF relay in Nakagami-m fading environment (positive integer fading index). In this case, the MGF of end-to-end SNR can be easily decomposed by partial fraction expansion as

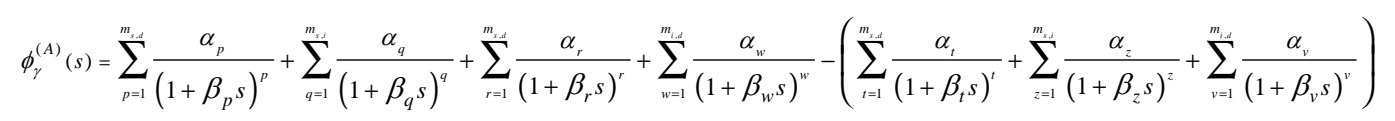

where

$$
\begin{aligned}
& \alpha_{p}=\frac{\left(-\frac{m_{s, i}}{m_{s, d}} \frac{\Omega_{s, i}}{\Omega_{s, d}}\right)^{m_{s, d}-p}}{\left(1-\frac{\Omega_{s, i}}{\Omega_{s, d}} \frac{m_{s, d}}{m_{s, i}}\right)^{m_{s, d}-p+1}}, \alpha_{q}=\frac{\left(-\frac{m_{s, i}}{m_{s, d}} \frac{\Omega_{s, d}}{\Omega_{s, i}}\right)^{m_{s, s}-q}}{\left(1-\frac{\Omega_{s, d}}{\Omega_{s, i}} \frac{m_{s, i}}{m_{s, d}}\right)^{m_{s, i}-q+1}}, \alpha_{r}=\frac{\left(-\frac{m_{i, d}}{m_{s, d}} \frac{\Omega_{i, d}}{\Omega_{s, d}}\right)^{m_{s, d}-r}}{\left(1-\frac{\Omega_{i, d}}{\Omega_{s, d}} \frac{m_{s, d}}{m_{i, d}}\right)^{m_{s, d}-r+1}}, \alpha_{w}=\frac{\left(-\frac{m_{i, d}}{m_{s, d}} \frac{\Omega_{s, d}}{\Omega_{i, d}}\right)^{m_{i, d}-w}}{\left(1-\frac{\Omega_{s, d}}{\Omega_{i, d}} \frac{m_{i, d}}{m_{s, d}}\right)^{m_{i, d}-w+1}},
\end{aligned}
$$

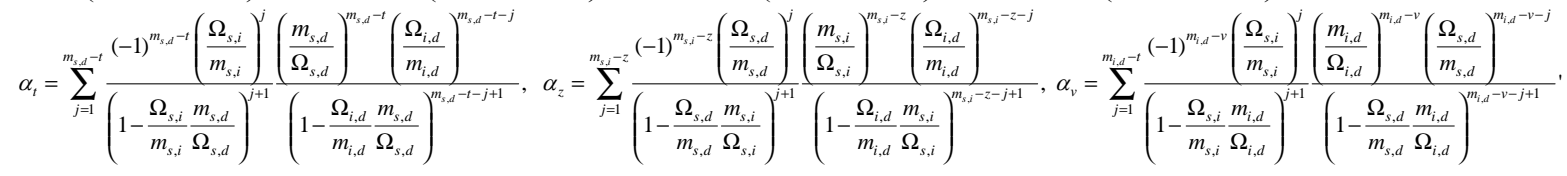

$$
\begin{aligned}
& \beta_{p}=\beta_{r}=\beta_{t}=\frac{\Omega_{s, d}}{m_{s, d}}, \beta_{q}=\beta_{z}=\frac{\Omega_{s, i}}{m_{s, i}} \text {, and } \beta_{w}=\beta_{v}=\frac{\Omega_{i, d}}{m_{i, d}} .
\end{aligned}
$$

Then it is not very difficult to derive its corresponding CDF (i.e., $P_{\text {out }}$ ) in closed-form, viz.,

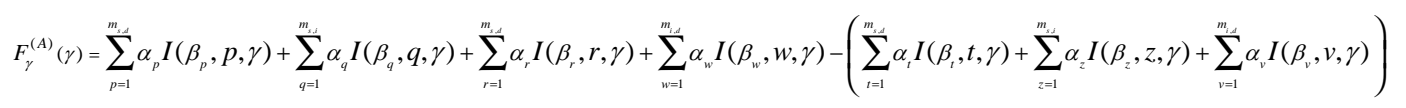

where $I(\sigma, k, \gamma)=\frac{G(k, \gamma / \sigma)}{\Gamma(k)}$ and $G(a, x)=\int_{0}^{x} t^{a-1} e^{-t} d t$ denotes the lower incomplete Gamma function.

Nevertheless, a numerical approach is recommended for the computation of the outage probability over generalized fading channel as it is much easier to program especially for multirelay networks.

Fig. 5 further demonstrates the utility and versatility of our proposed method for evaluating the outage probability of CAF relay networks in an i.n.d Rice fading environment. It is evident that our solution predicts the outage probability performance reasonably well over a wide range of SNRs and exhibits similar trends to that observed in Figure 3 and Figure 4 (i.e., our solution is much more accurate than using the MGF of lower and/or upper bound for end-to-end SNR). It is also important to highlight that most mathematical software packages (e.g., MATLAB, MAPLE, etc.) do not have a built-in generalized Marcum Q-function for its complex arguments. As a consequence, one could not directly evaluate the desired outage probability using a numerical Laplace or Fourier inversion technique (e.g., [32]-[33]]) with the closed-form MGF depicted in (11). We circumvent this difficulty by exploiting an alternative, rapidly converging series representation for the generalized $Q$-function in [34]. More importantly, our MGF formula (18) does not contain any special mathematical functions, and thus circumvents this problem all together. This example in turn highlights the usefulness of our approximation (18) to characterize the performance of CAF relay networks in many fading environments that heretofore had resisted simple solutions. 
International Journal of Computer Networks \& Communications (IJCNC) Vol.4, No.6, November 2012

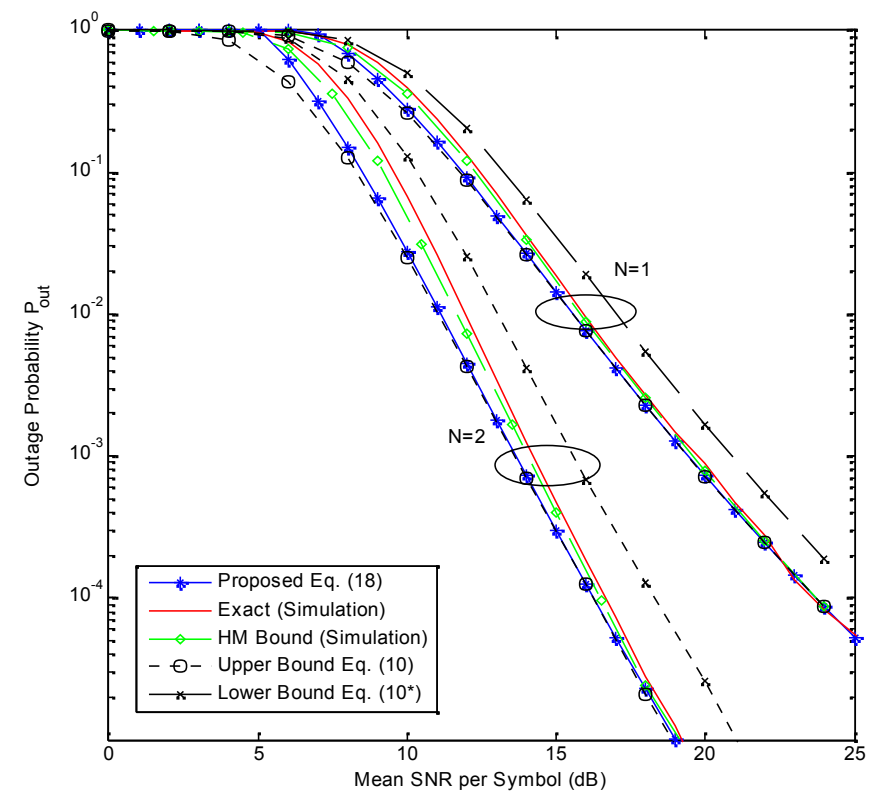

Fig. 5 Outage probability, $P_{\text {out }}$ of CAF relay networks for $\gamma_{t h}=5 \mathrm{~dB}$ over i.n.d Rice channels $(K=3)$ with $\Omega_{s, d}=0.2 \Omega_{T}, \Omega_{s, 1}=\Omega_{T}, \Omega_{s, 2}=0.3 \Omega_{T}, \Omega_{1, d}=0.8 \Omega_{T}$ and $\Omega_{2, d}=0.56 \Omega_{T}$.

\subsection{Ergodic Capacity of CAF Relay Networks with Limited CSI}

Our proposed MGF formula (18) can also be employed to compute the ergodic capacity of CAF relay networks more accurately and efficiently. Similar to [10], [12]-[13], we consider the problem of source adaptive transmission for 2-hop CAF multi-relay networks with limited CSI feedback in which rate at the source node is adapted according to prevailing channel condition (i.e., only the knowledge of the end-to-end SNR $\gamma_{T}$ at the destination node, and not all links is needed at the source node) while the relays simply amplify and forward the signals that they receive. However, the existing results were based on the upper and lower bounds for the MGF of $\gamma_{T}$. By exploiting a "desirable" exponential integral representation for ln (.) function [12], it is straight-forward to show that the normalized ergodic capacity (with respect to bandwidth $B$ ) of CAF relay networks is given by

$$
\frac{\bar{C}_{O R A}}{B}=\frac{1}{N+1} \frac{1}{\ln 2} \int_{0}^{\infty} \ln (1+\gamma) f_{\gamma}(\gamma) d \gamma=\frac{1}{N+1} \frac{1}{\ln 2} \int_{0}^{\infty} \frac{e^{-y}}{y}\left[1-\phi_{\gamma_{T}}(y)\right] d y
$$

Following the development similar to (22), it is also possible to evaluate (24) in closed-form for Rayleigh and Nakagami-m channels (positive integer $m$ ). For instance, it is not difficult to show that normalized ergodic capacity with a single cooperative relay over an i.n.d Nakagami-m channel is given by

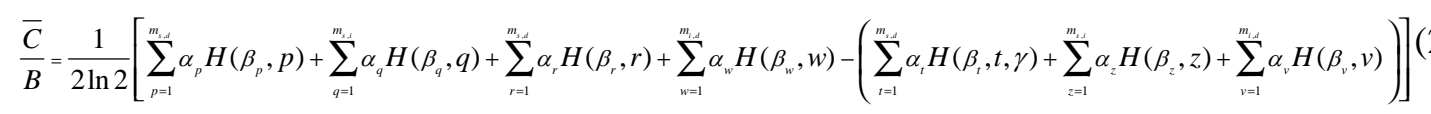

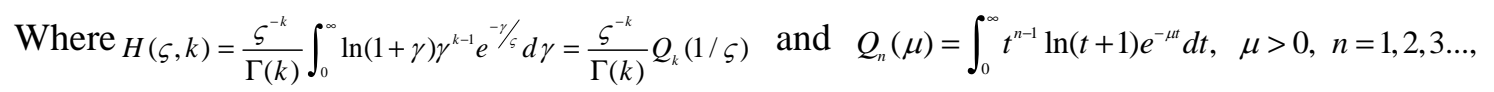
is an integral defined in [28, Eq. (15B.7)] which can be evaluated in closed-form as 
International Journal of Computer Networks \& Communications (IJCNC) Vol.4, No.6, November 2012

$Q_{n}(\mu)=\Gamma(n) e^{\mu} \sum_{\delta=1}^{n} \frac{\Gamma(-n+\delta, \mu)}{\mu^{\delta}}$. However, numerical evaluation of (24) (in conjunction with (18)) is recommended for its simplicity and applicability to a wide range of fading environments.

The usefulness of our approximate MGF (18) in the computation of the ergodic capacity is also demonstrated in Fig. 6 for a single CAF relay over an i.n.d Rayleigh fading channel. It is evident that our solution tightly approximates the exact performance curve for a wide range of mean link SNR, and considerably tighter than both the upper and lower bounds (i.e., Eq. (10) or Eq. (11)) considered in [10] and [12]. Thus, (24) (in conjunction with (18)) can be viewed both as an improvement and generalization of prior related works on ergodic capacity analysis for CAF relay networks.

\subsection{Link-Adaptive CAF Relay Networks}

Another important application of our MGF formula (18) is the joint-design of cooperative diversity with discrete-rate adaptive modulation. In fact, (18) could dramatically speed-up the evaluation of performance metrics of interest such as the achievable average spectral efficiency and the average bit error rate (ABER) for CAF multi-relay networks that employ adaptive modulation via the analytical framework employed in [22]-[25]. The average normalized spectral efficiency $\bar{C}_{A M}$ and ABER with adaptive modulation $\overline{B E R}_{A M}$ with $T$ modulation switching modes are given by (26) and (27), respectively:

$$
\begin{aligned}
& \bar{C}_{A M}=\sum_{n=1}^{T} \log _{2}\left(M_{n}\right) P_{n}=\sum_{n=1}^{T} n P_{n} \\
& \overline{B E R}_{A M}=\frac{\sum_{n=1}^{T} n P_{n} \overline{B E R_{n}}}{\sum_{n=1}^{T} n P_{n}}
\end{aligned}
$$

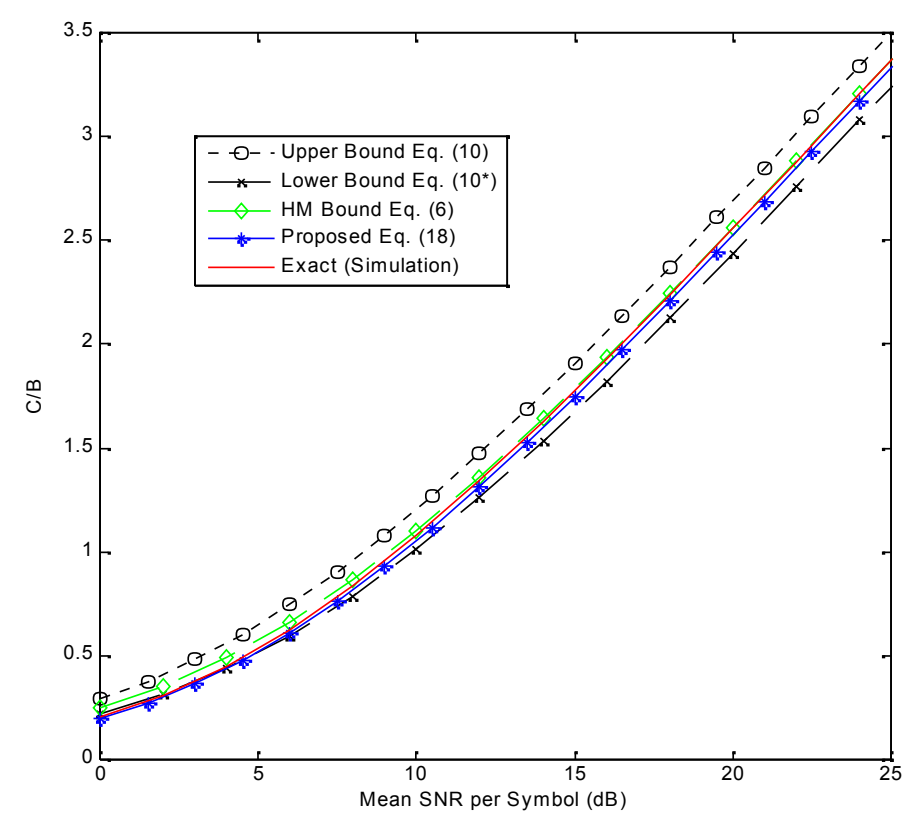

Fig. 6 Normalized ergodic capacity of single CAF relay networks over i.n.d Rayleigh fading channel with $\Omega_{s, d}=0.2 \Omega_{T}, \Omega_{s, 1}=\Omega_{T}, \Omega_{1, d}=0.5 \Omega_{T}$. 
International Journal of Computer Networks \& Communications (IJCNC) Vol.4, No.6, November 2012 where $P_{n}=\int_{\gamma_{n}}^{\gamma_{n+1}} f_{\gamma_{T}}(\gamma) d \gamma=F_{\gamma_{T}}\left(\gamma_{n+1}\right)-F_{\gamma_{T}}\left(\gamma_{n}\right)$ is the probability of selecting mode $n$ for transmission, $M_{n}=2^{n}$ corresponds to modulation constellation size during transmission mode $n$, , $\overline{B E R_{n}}=\frac{1}{P_{n}} \int_{\gamma_{n}}^{\gamma_{n+1}} B E R_{n}(\gamma) f_{\gamma_{T}}(\gamma) d \gamma$ is the average BER in mode $n$ while the CDF $F_{\gamma_{T}}(\gamma)$ can be evaluated numerically as in [32]-[33] using the MGF of $\gamma_{T}$ depicted in (18). Moreover, since the BER of MPSK with Gray coding can be closely approximated as $B E R_{n}=S E R_{n} / n[28$, Eq. (8.33)] (where $S E R_{n}$ denotes the symbol error rate of $M_{n}$-PSK), it is not difficult to show that $\overline{B E R_{n}}$ for discrete-rate M-PSK can be expressed as (using the CEP approximation in Table 1)

$$
\begin{gathered}
\overline{B E R_{n}}=\frac{1}{n P_{n}} \int_{\gamma_{n}}^{\gamma_{n+1}}\left[a_{1} \exp \left(-b_{1} \sin ^{2}\left(\pi / 2^{n}\right) \gamma\right)+c_{1} \exp \left(-2 b_{1} \sin ^{2}\left(\pi / 2^{n}\right) \gamma\right)\right] f_{\gamma_{T}}(\gamma) d \gamma \\
=\frac{1}{n P_{n}}\left\{a_{1}\left[\phi_{\gamma_{T}}\left(b_{1} \sin ^{2}\left(\pi / 2^{n}\right), \gamma_{n}\right)-\phi_{\gamma_{T}}\left(b_{1} \sin ^{2}\left(\pi / 2^{n}\right), \gamma_{n+1}\right)\right]\right. \\
\left.+c_{1}\left[\phi_{\gamma_{T}}\left(2 b_{1} \sin ^{2}\left(\pi / 2^{n}\right), \gamma_{n}\right)-\phi_{\gamma_{T}}\left(2 b_{1} \sin ^{2}\left(\pi / 2^{n}\right), \gamma_{n+1}\right)\right]\right\}
\end{gathered}
$$

where $\phi_{\gamma_{T}}(a, b)=\int_{b}^{\infty} \exp (-a \gamma) f_{\gamma_{T}}(\gamma) d \gamma$ is the marginal MGF of $\gamma_{T}$. As pointed out in [24]-[25], the marginal MGF of $\gamma_{T}$ can also be computed very efficiently (complexity similar to evaluating the CDF of $\gamma_{T}$ from its MGF) using the Laplace inversion technique [32]-[33] if the MGF of $\gamma_{T}$ is available in closed-form, which is true in our case (i.e., (18)). As pointed out in the preceding sections, it is also possible to derive a closed-form expression for the marginal MGF in Rayleigh and Nakagami-m fading environments using (18). Furthermore, the fixed switching thresholds $\gamma_{n}$ can be easily obtained for any arbitrary target instantaneous BER (i.e., $B E R_{T}$ ) by inverting the approximate BER formula (i.e., with the corresponding CEP expression in Table 1). For instance, the switching thresholds for the M-PSK modulation is given by

$$
\gamma_{n}=-\frac{1}{b_{1} \sin ^{2}\left(\pi / 2^{n}\right)} \ln \left(\frac{-a_{1}+\sqrt{a_{1}^{2}+4 n c_{1} B E R_{T}}}{2 c_{1}}\right)
$$

Sometimes, it is more appropriate to consider average packet error rate as in [24]-[25] (instead of ABER) for adaptive modulation especially for slow fading channels since the bit-to-bit independence assumption no longer holds and more importantly, adaptive modulation switching in practical systems cannot implemented at the "bit-level". Regardless of whether APER or ABER is used for performance comparison, our MGF expression (18) can always provide a more general and tractable solution with better accuracy than the upper or lower bound MGF of end-to-end SNR employed in earlier studies [24]-[25]. 


\section{Conclusion}

In this article, we have derived a novel approximate MGF of MRC combiner output SNR for multi-relay CAF networks in closed-form (i.e., Eq. (18)) that will facilitate efficient performance analysis of such networks over generalized fading channels (including composite-fading and mixed-fading scenarios). Numerical results indicate that our MGF expression is generally tighter than the widely used MGF of upper and lower bound of half harmonic mean SNR (even for the specific cases when a closedform solution for the latter is available) over a broad range of mean SNRs despite its simplicity and generality. Furthermore, our ASER expressions (also in closed-form) may be further exploited for system-level optimization such as finding the optimal power assignment among the cooperating nodes that minimizes the error rate, optimal relay placement, and so on. These extensions along with the application of the proposed approach in analysis of multi-hop multi-relay cooperative wireless networks is beyond the scope of this article but will be pursued as part of our future work.

\section{APPENDiX A}

[Proof of Lemma 1] The complementary cumulative CDF of $W=\frac{X Y}{X+Y+1}$ is given by

$$
\begin{aligned}
1-F_{W}(w) & =P\left[\frac{X Y}{X+Y+1}>w\right]=\int_{w}^{\infty}\left(\int_{\frac{w}{y+1)}}^{\infty} f_{X}(x) d x\right) f_{Y}(y) d y \\
& =\int_{w}^{\infty}\left[1-F_{X}\left(\frac{w(y+1)}{y-w}\right)\right] f_{Y}(y) d y
\end{aligned}
$$

Substituting $\int_{w}^{\infty} f_{Y}(y) d y=1-F_{Y}(w)$ in (A.1) and re-arranging the resulting expression, we get (14). This completes the proof.

\section{APPENDIX B}

[Proof of Lemma 2 and Eq. (17)] Noting that $\frac{w(y+1)}{y-w} \geq w$ for any non-negative $y>w$ and $F_{\kappa}($.$) is$ a monotonically increasing function (i.e., $F_{X}\left(\frac{w(y+1)}{y-w}\right) \geq F_{X}(w)$ ), it is straight forward to show to (14) can be lower bounded as

$$
F_{W}(w) \geq F_{Y}(w)+F_{X}(w) \int_{w}^{\infty} f_{Y}(y) d y
$$

which can be further simplified as

$$
F_{W}(w) \geq 1-\left[1-F_{Y}(w)\right]\left[1-F_{X}(w)\right]
$$

Expanding the product term of the right side of (B.2) and simplifying the resulting expression, we obtain (15). Also notice that the right side of (B.2) is simply the CDF of the first order statistics, viz., $Z=\min (X, Y)$. Thus the MGF of $Z$ can be determined by differentiating the right 
International Journal of Computer Networks \& Communications (IJCNC) Vol.4, No.6, November 2012

side of (B.2) with respect to $w$, and then evaluating the Laplace transform of the resulting expression, viz.,

$$
\phi_{Z}(s)=\phi_{X}(s)+\phi_{Y}(s)-\int_{0}^{\infty} e^{-s w}\left[f_{X}(w) F_{Y}(w)+f_{Y}(w) F_{X}(w)\right] d w
$$

Interestingly, the last term of (B.3) resembles the MGF of output SNR for a dual diversity selection combining, and therefore (B.3) can be re-stated as

$$
\phi_{W}(s) \geq \phi_{Y}(s)+\phi_{X}(s)-\phi_{\gamma_{S D C}}(s)
$$

Next, recognizing that $\phi_{\gamma_{S D C}}(s)>\phi_{\gamma_{M R C}}(s)$, we can tightly approximate (B.4) as

$$
\phi_{W}(s) \approx \phi_{Y}(s)+\phi_{X}(s)-\phi_{X}(s) \phi_{Y}(s)
$$

since $\phi_{\gamma_{M R C}}(s)=\phi_{X}(s) \phi_{Y}(s)$. This completes the proof of (17).

\section{ACKNOWLEDGEMENTS}

This work was supported in part by funding from the US Army Research Office (W911NF-042-0054), Clarkson Aerospace, and the National Science Foundation (0931679 and 1040207).

\section{REFERENCES}

[1] N. Laneman, D. Tse, and G. Wornell, "Cooperative Diversity in Wireless Networks: Efficient Protocols and Outage Behaviour," IEEE Trans. Info. Theory, vol. 50, pp. 3062-3080, Dec. 2004.

[2] M. A. Khojastepour, A. Sabharwal, and B. Aazhang, "Lower Bounds on the Capacity of Gaussian Relay Channel," Proc. CISS'04, Princeton, pp. 597-602, Mar. 2004.

[3] A. Bletsas, A. Khisti, D. P. Reed, and A. Lippman, "A Simple Cooperative Diversity Method based on Network Path Selection,” IEEE J. Sel. Areas Commun., vol. 24, March, 2006.

[4] M. O. Hasna and M. S. Alouini, "A Performance Study of Dual-hop Tranmission with Fixed Gain Relays”, IEEE Trans. Wireless Commun., vol. 3, pp. 1963-1968, Nov. 2004.

[5] M. Hasna, M. S. Alouini, "End-to-End Performance of Transmission Systems with Relays over Rayleigh- $\quad$ Fading Channels," IEEE Trans. Wireless Commun., vol. 2, pp. 1126-1131, Nov. 2003.

[6] P. Anghel, M. Kaveh "Exact Symbol Error Probability of a Cooperative Network in a RayleighFading Environment," IEEE Trans. Wireless Commun., vol. 3, pp. 1416-1421, Sep. 2004.

[7] Weifeng Su, K. S. Ahmed and K. J. Ray Liu, "Cooperative Communication Protocols in Wireless Networks: $\quad$ Performance Analysis and Optimum Power Allocation," Springer Journal on Wireless Personal Communications, vol. 44, pp. 181-217, 2008.

[8] M. Hasna, M. Alouini, "Harmonic Mean and End-to-End Performance of Transmission Systems With Relays," IEEE Trans. Communications, vol. 52, pp. 130-135, Jan. 2004.

[9] A Ribeiro, X. Cai, G. Giannakis "Symbol Error Probabilities for General Cooperative Links," IEEE Trans. Wireless Communications, vol. 4, pp. 1264-1273, May 2005.

[10] T. Nechiporenko, K. Phan, C. Tellambura, and H. H. Nguyen, "On the Capacity of Rayleigh Fading Cooperative Systems under Adaptive Transmission," IEEE Trans. Wireless Communications, vol. 8, pp. 1626- 1631, April 2009.

[11] S. Ikki and M. Ahmed, "Performance Analysis of Cooperative Diversity Wireless Networks over Nakagami-m Fading Channels," IEEE Communication Letters, vol. 11, pp. 334-336, April 2007. 
International Journal of Computer Networks \& Communications (IJCNC) Vol.4, No.6, November 2012

[12] A. Annamalai, B. Modi, R. Palat and J. Matyjas, "Tight Bounds on the Ergodic Capacity of Cooperative Analog Relaying with Adaptive Source Transmission Techniques," Proc. IEEE PIMRC'10, pp. 18-23, 2010.

[13] B. C. Modi, O. Olabiyi and A. Annamalai, "On Ergodic Capacity of Cooperative Amplify-andForward Relay Networks in Rice Fading Environments," Proc. IEEE GLOBECOM HeterWMN Workshop, Dec. 2011.

[14] D. Senaratne and C. Tellambura, "Unified Exact Performance Analysis of Two-Hop Amplify-andForward Relaying in Nakagami Fading," IEEE Trans. Vehicular Technology, vol. 59, pp. 15291534, March 2010.

[15] B. Maham and A. Hjørungnes, "Asymptotic Performance Analysis of Amplify-and-Forward Cooperative Networks in a Nakagami-m Fading Environment," IEEE Communications Letters, pp. 300-302, May 2009.

[16] M. Di Renzo, F. Graziosi, and F. Santucci, "A Unified Framework for Performance Analysis of CSI-Assisted Cooperative Communications over Fading Channels," IEEE Trans. Communications, vol. 57, pp. 2551-2557, Sept. 2009.

[17] A. Annamalai, O. Olabiyi, and S. Alam, "Accurate Approximations of Error Rates for Cooperative Non- Regenerative Relay Systems over Generalized Fading Channels," Proc. IEEE IWCMC'11, pp. 906911, July 2011.

[18] R. H. Y. Louie, Y. Li, and B. Vucetic, "Performance Analysis of Beamforming in Two-hop Amplify-and- $\quad$ Forward Relay Networks," Proc. IEEE ICC, pp. 4311-4315, May 2008.

[19] O. Olabiyi and A. Annamalai, "ASER Analysis of Cooperative Non-Regenerative Relay Systems over Generalized Fading Channels," Proc. IEEE ICCCN, August, 2011.

[20] T. Nechiporenko, P. Kalansuriya, and C. Tellambura, "Performance of Optimum Switching Adaptive M-QAM for Amplify-and-Forward Relays," IEEE Trans. Vehicular Technology, vol. 58, pp. 2258-2268, June 2009.

[21] T. Nechiporenko, K. Phan, C. Tellambura and H. Nguyen, "Performance Analysis of Adaptive MQAM for Rayleigh Fading Cooperative Systems," Proc. IEEE ICC, pp. 3393-3399, May 2008.

[22] B. Modi and A. Annamalai, "Improving the Spectral Efficiency of Amplify-and-Forward Cooperative Relay Network with Adaptive M-QAM Modulation," Proc. IEEE ICCCN, Aug.

2011.

[23] A. Annamalai , B. Modi, and R. C. Palat, "Analysis of Cooperative Non-regenerative Relay Networks with Adaptive Modulation in Generalized Fading Channels," Proc. IWCMC, pp. 760-765, July 2011.

[24] B. C. Modi, O. Olabiyi, and A. Annamalai, "Improving the Spectral Efficiency of Adaptive Modulation in Amplify-and-Forward Cooperative Relay Networks with Truncated ARQ Protocol," Proc. IEEE GLOBECOM'11, Houston, Dec. 2011.

[25] B. C. Modi, O. Olabiyi, and A. Annamalai, "Joint-Design of Adaptive Modulation and Coding with ARQ for Cooperative Relay Networks," Proc. IEEE MILCOM, Baltimore, Nov. 2011.

[26] Z. Wang and G. Giannakis, "A Simple and General Parameterization Quantifying Performance in Fading Channels," IEEE Trans. Communications, vol. 51, pp. 1389-1398, Aug. 2003.

[27] Y. Ma, Z. Wang, and S. Pasupathy, "Asymptotic Performance of Wireless Communications with Generalized Selection Combining," Proc. IEEE GLOBECOM'03, pp. 1679-1683, Dec. 2003.

[28] M. K. Simon and M. S. Alouini, Digital Communication over Fading Channels, $2^{\text {nd }}$ ed., John Wiley \& Sons, 2005. 
International Journal of Computer Networks \& Communications (IJCNC) Vol.4, No.6, November 2012

[29] I. Gradshteyn and I. Ryzhik, Table of Integrals, Series and Products, $5^{\text {th }}$ ed., Academic Press, 1994.

[30] M. Chiani, D. Dardari, and M. K.Simon, "New Exponential Bounds and Approximations for the Computation of Error Probability in Fading Channels," IEEE Trans. Wireless Commun., vol. 2, pp. 840-845, July 2003.

[31] J. W. Craig, "A New, Simple and Exact Result for Calculating the Probability of Error for TwoDimensional Signal Constellations," Proc. IEEE MILCOM, May 1991.

[32] R. Palat, A. Annamalai and J. Reed, "An Efficient Method for Evaluating Information Outage Probability and Ergodic Capacity of OSTBC Systems," IEEE Commun. Letters, vol. 12, pp. 191-193.

[33] Y. C. Ko, M. S. Alouini, and M. K. Simon, "Outage Probability of Diversity Systems over Generalized Fading Channels," IEEE Trans. Communications, vol. 48, Nov. 2000.

[34] A. Annamalai, O. Olabiyi, S. Alam, O. Odejide, and D. Vaman, "Unified Analysis of Energy Detection of Unknown Signals over Generalized Fading Channels," Proc. IEEE IWCMC, July 2011.

Oluwatobi O. Olabiyi received the B.Sc. degree in Electronic and Electrical Engineering from Obafemi Awolowo University, Ile-Ife, Nigeria, and the M.S. degree in Electrical Engineering Prairie View A\&M University, Texas, USA. Over the last two years, he has co-authored approximately two-dozen peer-reviewed conference and journal articles. He was the recipient of the Roy G. Perry College of Engineering Outstanding Masters Student of the Year Award (2011) and the National Society of Black Engineer's Golden Torch Award for Graduate Student of Year (2012). He is presently continuing his doctoral studies at the Prairie View A\&M University. His research interests include dynamic spectrum access, MIMO, cooperative communications, statistical signal processing, compressive sensing, machine-learning and optimization techniques.

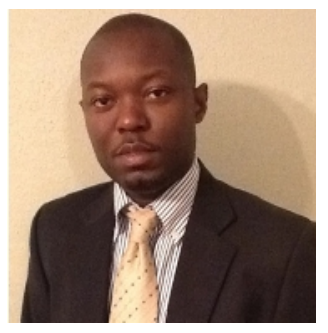

Dr. Annamalai is presently the Director of Center of Excellence for Communication Systems Technology Research, a Texas A\&M Board of Regents approved University Research Center at the Prairie View A\&M University, and a tenured faculty member in the Department of Electrical and Computer Engineering. He has over 16 years of research/teaching experience in wireless communications at Motorola, University of Victoria, Air Force Research Laboratory, Virginia Tech and PVAMU with approximately 200 peer-reviewed publications and 5 book chapters. Dr. Annamalai has been honored by his colleagues on numerous occasions for his excellence in research including winning the 2011 Roy G. Perry College of Engineering Outstanding Faculty (Research) Award,

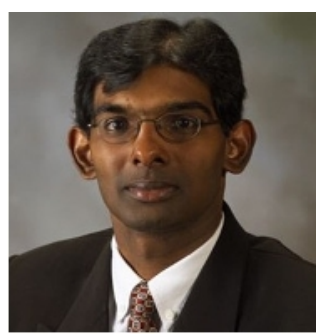
IEEE Leon Kirchmayer Prize Paper award, ASEE/AFOSR Summer Faculty Fellowships, NSERC Doctoral Prize, CAGS/UMI Distinguished Doctoral Dissertation Award, IEEE VTS/Motorola Daniel E. Noble Fellowship, among others. He had served on the Editorial Boards of four IEEE journals/transactions in the last 12 years, and has helped to organize a few major IEEE conferences on wireless communications including serving in the capacity of Technical Program Chair of the 2002 IEEE Vehicular Technology Conference in Vancouver, Canada. His current research interests include cooperative spectrum sensing, compressive sensing, cross-layer design for scalable multimedia transmission and cooperative wireless communications. 